\title{
Caracterización clínica y epidemiológica de los pacientes con COVID-19 en un hospital situado en la altura
}

Aníbal Díaz-Lazo* 1,2,a,b; Raul Montalvo Otivo 1,3,c; Ernesto Lazarte Nuñez 1,4,a; Edinson Aquino Lopez ${ }^{1, \mathrm{~d}}$

\section{RESUMEN}

Objetivo: Determinar las características clínicas y epidemiológicas de los pacientes con COVID-19 en un hospital situado en la altura.

Materiales y métodos: Estudio de diseño observacional, transversal y analítico realizado en el Hospital Daniel Alcides Carrión de Huancayo, Perú, ubicado a 3250 m s. n. m. La población estuvo conformada por 2248 personas. El diagnóstico de COVID-19 fue realizado con la prueba serológica y/o la prueba de reacción en cadena de la polimerasa transcriptasa inversa (RT-PCR). Los datos se registraron en un cuestionario. Para el análisis estadístico se emplearon las pruebas de $\mathrm{X}^{2}$ y $\mathrm{t}$ de Student, y la regresión logística bivariada y multivariada. El valor de $p<0,05$ fue considerado significativo.

Resultados: La población con diagnóstico de COVID-19 fue de 760 pacientes (100,00\%). De ellos, los hombres fueron los más afectados con el 62,36 \% (474 personas). El promedio de edad fue de 49 años (rango: 15 a 95) y el mayor número de casos se encontró en el grupo etario de 40 a 59 años (38,82 \%), sobre todo entre las amas de casa (21, $44 \%)$ y los comerciantes $(19,21 \%)$. Los síntomas más comunes de la COVID-19 fueron tos (70,26 \%), malestar general (57,63 \%), dificultad respiratoria $(48,81 \%)$, fiebre $(48,15 \%)$ y dolor de garganta $(44,21 \%)$. Entre las comorbilidades más frecuentes encontramos obesidad $(4,47 \%)$, diabetes mellitus $(2,76 \%)$ e hipertensión arterial $(1,31 \%)$. El $51,71 \%$ de los pacientes requirió hospitalización y la frecuencia de letalidad fue de $14,21 \%$. Los factores asociados a un riesgo de mortalidad fueron la edad de 60 años a más (OR = 4,74; IC 95\%: 2,94-7,65; $p=0,000)$, la dificultad respiratoria $(\mathrm{OR}=2,38$; IC 95\%: 1,42-3,99; $p=0,001)$ y el uso de ventilación mecánica $(\mathrm{OR}=8,75 ; \mathrm{IC} 95 \%: 4,45-17,22 ; p=0,000)$. Conclusiones: La COVID-19 afecta con mayor frecuencia a la población adulta. La tos y el malestar general fueron los síntomas más comunes, mientras que la edad avanzada, la disnea y el uso de ventilación mecánica se asociaron a mayor riesgo de letalidad.

Palabras clave: Infecciones por coronavirus; Epidemiologia; Mortalidad; Hipertensión (Fuente : DeCS BIREME).

\section{Clinical and epidemiological characteristics of patients with COVID-19 at a high-altitude hospital}

\section{ABSTRACT}

Objective: To determine the clinical and epidemiological characteristics of patients with COVID-19 at a high-altitude hospital.

Materials and methods: An observational, cross-sectional and analytical study carried out at the Hospital Daniel Alcides Carrión in Huancayo, Peru, located at 3,250 m a.s.l. The population consisted of 2,248 people. COVID-19 was diagnosed through serology testing and/or reverse transcription polymerase chain reaction (RT-PCR) testing. Data were recorded in a questionnaire. For the statistical analysis, the $\mathrm{X}^{2}$ test, Student's t-test, and bivariate and multivariate logistic regression analyses were used. A $p$ value $<0.05$ was considered statistically significant.

1 Hospital Regional Docente Clínico Quirúrgico Daniel A. Carrión, Departamento de Medicina. Huancayo, Perú.

2 Universidad Peruana Los Andes de Huancayo, Facultad de Medicina Humana. Perú.

3 Universidad Nacional del Centro del Perú, Facultad de Medicina. Huancayo, Perú.

4 Universidad Continental de Huancayo, Facultad de Medicina Humana. Huancayo, Perú.

a Médico Internista.

b Doctor en Medicina.

c Medico Infectólogo.

d Médico Cirujano.

*Autor corresponsal. 
Results: The study population with a diagnosis of COVID-19 accounted for 760 patients (100 \%), most of whom were males (474 individuals; $62.36 \%$ ). The average age was 49 years (range: 15 to 95). Most cases were found in the age group of 40 to 59 years $(38.82 \%)$, particularly among housewives $(21.44 \%)$ and traders $(19.21 \%)$. The most common COVID-19 symptoms were cough $(70.26 \%)$, malaise $(57.63 \%)$, respiratory distress $(48.81 \%)$, fever $(48.15 \%)$ and sore throat $(44.21 \%)$. The most frequent comorbidities were obesity $(4.47 \%)$, diabetes mellitus $(2.76 \%)$ and arterial hypertension (1.31\%). Fifty-one point seven one percent $(51.71 \%)$ of the patients required hospitalization and the mortality rate was $14.21 \%$. The risk factors for mortality were age 60 years or older $(\mathrm{OR}=4.74 ; 95 \% \mathrm{Cl}: 2.94-7.65 ; p=0.000)$, respiratory distress $(\mathrm{OR}=2.38$; $95 \% \mathrm{Cl}: 1.42-3.99 ; p=0.001)$ and use of mechanical ventilation $(\mathrm{OR}=8.75 ; 95 \% \mathrm{Cl}: 4.45-17.22 ; p=0.000)$.

Conclusions: COVID-19 most frequently affects the adult population. Cough and malaise were the most common symptoms, while older age, dyspnea and use of mechanical ventilation were associated with a higher risk of mortality.

Keywords: Coronavirus infections; Epidemiology; Mortality; Hypertension (Source: MeSH NLM).

\section{INTRODUCCIÓN}

La enfermedad por coronavirus 2019 (COVID-19) es causada por el coronavirus 2 del síndrome respiratorio agudo severo (SARS-CoV-2). Desde el reporte del primer caso, la COVID-19 se ha diseminado rápidamente por varios países y se ha convertido en una emergencia de salud pública. El 11 de marzo del 2020, la Organización Mundial de la Salud (OMS) la declaró una pandemia (1-3). El SARS-CoV-2 ha sido considerado como el virus que tiene el mayor riesgo de infección y de fallecimiento en todo el mundo ${ }^{(1,2)}$, lo cual está relacionado a su forma de transmisión entre las personas: a través de gotitas aerosolizadas y por el contacto con superficies contaminadas ${ }^{(4,5)}$. Los factores relacionados con el control de la propagación de la enfermedad se refieren, sobre todo, al distanciamiento físico, al uso de mascarilla y a una higiene adecuada de las manos ${ }^{(3-5)}$.

Los principales síntomas de la COVID-19 son fiebre, tos seca, fatiga, mialgia y disnea leve, y con menor frecuencia cefalea, dolor de garganta, congestión nasal, náusea, vómitos y diarrea ${ }^{(6,7)}$. Entre el 80 y $90 \%$ de las personas desarrollan cuadros leves; en el $10 \%$, los casos son moderados y se manifiestan con disnea, hipoxemia y con compromiso pulmonar mayor del $50 \%$; y en $5 \%$, los casos pueden ser graves y cursar con neumonía, insuficiencia respiratoria, shock, falla multiorgánica, trastornos de la coagulación, y llegar a la muerte ${ }^{(4,7)}$. Los resultados de los exámenes para diagnosticar la COVID-19, tales como las pruebas serológicas, la prueba de reacción de la polimerasa transcriptasa reversa (RT-PCR, del inglés Reverse transcription polymerase chain reaction), deben interpretarse de acuerdo con los factores epidemiológicos, clínicos y radiológicos (4). Hasta la fecha, no existe un tratamiento eficaz para la COVID-19 ${ }^{(3)}$. El índice de letalidad es variable (entre $2 \%$ y $12,8 \%$ ) y puede incrementarse con el aumento de la edad y la presencia de comorbilidades ${ }^{(4,8,9)}$.

Existen múltiples factores asociados a complicaciones por COVID-19, como la edad de 65 años a más, obesidad, hipertensión arterial, diabetes tipo 2, asma moderada a grave, las enfermedades cardiovasculares severas, enfermedad pulmonar crónica, insuficiencia renal crónica y enfermedad o tratamiento inmunosupresor ${ }^{(10,11)}$.

Existen pocos estudios realizados sobre la infección por el SARS-CoV-2 en ciudades situadas en zonas de altitud y en su población nativa, la que ha desarrollado cambios fisiológicos adaptativos para sobrevivir en un ambiente con niveles de presión atmosférica baja y con valores de saturación de oxígeno $\left(\mathrm{SatO}_{2}\right)$ menores en comparación con los habitantes de ciudades ubicadas a nivel del mar. Es conocido que el SARS-CoV-2 afecta los pulmones, en primer lugar, y con menor frecuencia, los demás órganos. En estas condiciones, es posible que pudiera existir variabilidad en los aspectos epidemiológicos, en la presentación clínica, en las variables sociodemográficas, etc. En ese contexto, se diseñó un estudio con el objetivo de determinar las características clínicas y epidemiológicas de los pacientes con COVID-19 que viven en una zona de altura y que acuden a un hospital público.

\section{MATERIALES Y MÉTODOS}

\section{Diseño y población de estudio}

Estudio de tipo observacional, transversal y analítico realizado en el Hospital Regional Docente Clínico Quirúrgico Daniel Alcides Carrión (HRDCQ DAC) de Huancayo, del 27 de marzo al 31 de julio del 2020. La población (N) fue de 2248 personas, quienes fueron evaluadas para diagnosticar COVID-19. Se aplicaron criterios de inclusión y exclusión para determinar a los pacientes portadores de la enfermedad. El criterio de inclusión fue ser un paciente con prueba serológica y/o prueba molecular (PCR-RT) positiva para COVID-19 atendido en el hospital RDCQ DAC. Se excluyeron a los pacientes que procedían de lugares ubicados en zonas de altura menores que $3000 \mathrm{~m} \mathrm{~s}$. n. m., de la selva o de la costa, y también los registros con datos incompletos. La muestra final fue de 760 pacientes con diagnóstico positivo de COVID-19.

\section{Variables y mediciones}

Las variables que intervienen en la categorización fueron 
sociodemográficas (edad, sexo, ocupación, procedencia), el antecedente de contacto, los síntomas y signos, hospitalización, comorbilidades, uso de ventilación mecánica y evolución.

El diagnóstico para un caso confirmado de COVID-19 se define como una persona asintomática o con infección respiratoria aguda que presenta tos o dolor de garganta y al menos uno o más de los siguientes síntomas y signos: fiebre, cefalea, dificultad para respirar y congestión nasal; y que tiene una prueba de laboratorio positiva para COVID-19, ya de reacción de la polimerasa transcriptasa reversa (RT-PCR) tomada de muestra nasofaríngea y orofaríngea $\mathrm{y} / \mathrm{o}$ una prueba rápida de detección de $\lg M, \lg G$ o $\lg G / \lg M{ }^{(10)}$. Un contacto previo es una persona que ha estado en contacto durante los dos días anteriores y los 14 días posteriores a la aparición de síntomas con un caso probable o confirmado de COVID-19 ${ }^{(12,13)}$. La defunción por COVID-19 es la muerte resultante de una enfermedad clínicamente compatible en un caso confirmado de COVID-19 (12).

La técnica de recolección de datos empleada fue el análisis documental, y como instrumento se utilizó un cuestionario denominado ficha de investigación clínica epidemiológica aplicado al ingreso al hospital para la atención médica y el seguimiento correspondiente. Se registraron los datos de las variables de estudio y el resultado de la prueba diagnóstica serológica y/o RT-PCR para COVID-19.

\section{Análisis estadístico}

Las variables categóricas se expresaron en frecuencias y porcentajes; y las variables numéricas, en promedios y desviación estándar. Para el análisis inferencial de los datos se emplearon las pruebas de $\mathrm{X}^{2}$ (para las variables categóricas,) y t de Student, (para las variables continuas por tener los datos una distribución normal). La asociación fue determinada con la logística bivariada y multivariada y para obtener la fuerza de asociación se utilizó el Odds ratio (OR) con intervalo de confianza al 95\% (IC95\%). El valor de $p<0,05$ fue considerado significativo.

\section{Consideraciones éticas}

Este trabajo fue aprobado por el Comité de Ética en Investigación del HRDCQ DAC de Huancayo. Durante el desarrollo del estudio se han respetado los principios bioéticos de investigación en humanos. Tanto el procesamiento como el análisis de datos mantuvieron la reserva y la confidencialidad del caso.

\section{RESULTADOS}

De 2248 (100\%) personas que fueron evaluadas para diagnosticar COVID-19, 760 (33,81\%) presentaron los criterios de diagnóstico (promedio de edad $=49,00 \pm 15,95$ años y rango: 14 y 95$)$ y $1488(66,19 \%)$ personas no lo presentaron (promedio de edad $=41,64 \pm 14,93$ años, y rango: 10 y 95) $(p=0,000)$. En el grupo de las 760 personas afectadas por la COVID-19 (100,00\%), el 62,36\% (474) fueron hombres y el $37,64 \%$ (286), mujeres, en una proporción hombre/mujer de 1,65 . Mientras que en el grupo de personas sin diagnóstico de COVID-19, que fueron $1488(100,00 \%)$, el $56,65 \%$ (843) fueron mujeres y el $43,35 \%$ (645), hombres.

En la figura 1 se muestra que el porcentaje de casos de la COVID-19 (66,32\%) es mayor a partir de los 40 años de edad, sobre todo en el grupo etario entre 40 y 59 años $X^{2}$ (38,82\%). Además, entre los 60 y 79 años se encontró un mayor porcentaje de casos confirmados de COVID-19 (24,21\%) que de personas sanas $(11,97 \%)(p=0,000)$, mientras que en el grupo etario entre los 20 y 39 años de edad, hallamos más personas que no tuvieron la enfermedad frente a las que sí se infectaron $(49,97 \%$ vs. $32,63 \% ; p=0,000)$.

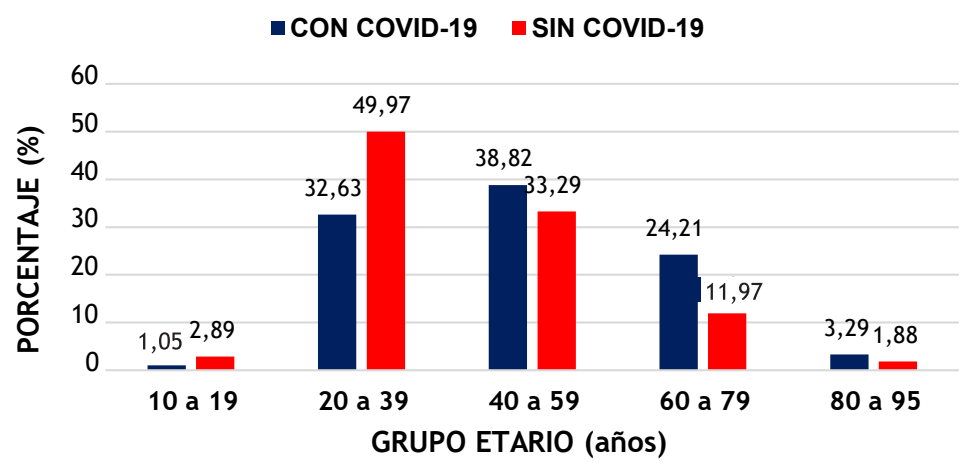

Figura 1. Frecuencia de COVID-19 según grupo etario

El 86,00 \% de las personas con COVID-19 provenían del cercado de Huancayo $(49,25 \%)$, de los distritos de $\mathrm{El}$ Tambo $(19,73 \%)$, Chilca $(17,36 \%)$ y de otros distritos y provincias $(13,66 \%)$ : todos los lugares están ubicados a más de $3000 \mathrm{~m} \mathrm{~s}$. $\mathrm{n}$. m. En la tabla 1 se muestra la frecuencia de personas afectadas por COVID-19 
de acuerdo a su ocupación. El 40,65 \% de los casos corresponde a las amas de casa $(21,44 \%)$ y comerciantes $(19,21 \%)$. A continuación, el grupo que labora como conductor, chofer o transportista de vehículos $(8,68 \%)$; en cuarto lugar, los trabajadores de los establecimientos de salud $(8,28 \%)$, seguidos de los agricultores $(3,55 \%)$. En menor frecuencia se contagiaron los militares $(2,36 \%)$ y los policías $(2,36 \%)$.

Tabla 1. Frecuencia de contagio de la COVID-19 según ocupación

\begin{tabular}{|lrr|}
\multicolumn{1}{c}{ Ocupación } & \multicolumn{2}{c}{$\mathrm{n=760}$} \\
& N & (\%) \\
\hline Ama de casa & 163 & 21,44 \\
\hline Comerciante & 146 & 19,21 \\
\hline Conductor, chofer o transportista & 66 & 8,68 \\
\hline Trabajador de salud & 63 & 8,28 \\
\hline Agricultor & 27 & 3,55 \\
\hline Albañil & 21 & 2,76 \\
\hline Estudiante & 19 & 2,50 \\
\hline Militar & 18 & 2,36 \\
\hline Policía Nacional & 18 & 2,36 \\
\hline
\end{tabular}

En el grupo con COVID-19, se investigó sobre el lugar en el que hubo un contacto previo con un caso confirmado o sospechoso de COVID-19, por ser el posible foco de contagio, y encontramos que el $18,28 \%$ (139) de los casos ocurrió en el entorno familiar y el 10,26 \% (78), en el trabajo; en el 71,46\% (543), no se estableció si existió un contacto previo con caso de COVID-19. La tabla 2 muestra que los síntomas más comunes fueron tos $(70,26 \%)$, malestar general $(57,63 \%)$, fiebre $(48,81 \%)$, dificultad respiratoria $(48,15 \%)$ y dolor de garganta $(44,21 \%)$. También observamos síntomas digestivos como diarrea $(11,31 \%)$, náuseas y/o vómitos $(9,21 \%)$ y dolor abdominal $(4,07 \%)$ en menor porcentaje.

Tabla 2. Síntomas en pacientes con COVID-19

\begin{tabular}{|lrr|}
\hline \multicolumn{1}{c}{ Síntomas } & \multicolumn{2}{c}{$\mathrm{n}=760$} \\
& $\mathrm{~N}$ & $(\%)$ \\
\hline Tos & 534 & 70,26 \\
\hline Malestar general & 438 & 57,63 \\
\hline Dificultad respiratoria & 371 & 48,81 \\
\hline Fiebre & 366 & 48,15 \\
\hline Dolor de garganta & 336 & 44,21 \\
\hline Cefalea & 201 & 26,44 \\
\hline Dolor muscular & 180 & 23,68 \\
\hline Dolor de pecho & 152 & 20,00 \\
\hline Congestión nasal & 130 & 17,10 \\
\hline Diarrea & 86 & 11,31 \\
\hline Náuseas y/o vómitos & 70 & 9,21 \\
\hline Dolor de articulaciones & 37 & 4,86 \\
\hline Dolor abdominal & 31 & 4,07 \\
\hline Irritabilidad y/o confusión & 16 & 2,10 \\
\hline
\end{tabular}

En la tabla 3 se muestra que las comorbilidades más frecuentes en los pacientes con COVID-19 fueron la obesidad $(4,47 \%)$, diabetes mellitus $(2,76 \%)$ e hipertensión arterial $(1,31 \%)$.
Las enfermedades respiratorias $(1,30 \%)$ y cardiovasculares crónicas $(0,78 \%)$ se presentaron en frecuencias bajas. 
Tabla 3. Comorbilidades en pacientes con COVID-19

\begin{tabular}{|lrr|}
\multicolumn{1}{c}{ Comorbilidades } & \multicolumn{2}{c}{$\mathrm{n=760}$} \\
& $\mathrm{N}$ & $(\%)$ \\
\hline Obesidad & 34 & 4,47 \\
\hline Diabetes mellitus & 21 & 2,76 \\
\hline Hipertensión arterial & 10 & 1,31 \\
\hline Enfermedad cardiovascular crónica & 6 & 0,78 \\
\hline Asma bronquial & 5 & 0,65 \\
Antecedente de tuberculosis pulmonar & 5 & 0,65 \\
\hline Artrosis & 4 & 0,52 \\
\hline
\end{tabular}

En el 48,02 \% de los casos (365 pacientes) se indicó autoaislamiento en casa por no tener manifestaciones clínicas; en el 51,98 \% (395), los pacientes fueron hospitalizados por presentar síntomas y gravedad moderada a severa. En el 7,36 \% (56) el estado de gravedad era crítico, por lo que requirió manejo en la Unidad de Cuidados Intensivos y uso de ventilación mecánica. Un grupo de pacientes falleció en la Emergencia. En esta serie se encontró una frecuencia de letalidad del 14,21\%. El $69,44 \%$ (75) fueron hombres, con una relación de 2,2 en comparación con las mujeres. El promedio de edad para los fallecidos fue mayor $(60,82 \pm 14.16$ años), frente a la de los sobrevivientes $(46,96 \pm 15,33$ años $)(p<0,000)$. En la tabla 4, según el análisis univariado, se muestra que la edad de 60 años a más, la dificultad respiratoria, la diarrea, la obesidad y el uso de terapia de ventilación mecánica estuvieron asociados a mayor frecuencia de mortalidad, mientras que la fiebre se consideró como un factor protector.

Tabla 4. Factores asociados en pacientes fallecidos con COVID-19 (análisis univariado)

\begin{tabular}{|c|c|c|c|}
\hline Variable & $\begin{array}{l}\text { Odds ratio } \\
\text { (OR) }\end{array}$ & IC a $95 \%$ & Valor de $p$ \\
\hline \multicolumn{4}{|l|}{ Aspectos demográficos } \\
\hline Edad $>60$ años & 4,74 & $2,94-7,65$ & 0,000 \\
\hline Sexo masculino & 1,59 & $0,96-2,64$ & 0,071 \\
\hline \multicolumn{4}{|l|}{ Síntomas } \\
\hline Tos & 0,87 & $0,49-1,53$ & 0,633 \\
\hline Malestar general & 0,89 & $0,53-1,49$ & 0,681 \\
\hline Fiebre & 0,38 & $0,23-0,64$ & 0,000 \\
\hline Dificultad respiratoria & 2,38 & $1,42-3,99$ & 0,001 \\
\hline Dolor de garganta & 0,96 & $0,57-1,61$ & 0,886 \\
\hline Cefalea & 0,87 & $0,49-1,56$ & 0,660 \\
\hline Dolor muscular & 0,76 & $0,40-1,43$ & 0,397 \\
\hline Dolor de pecho & 1,13 & $0,59-2,15$ & 0,706 \\
\hline Congestión nasal & 1,24 & $0,63-2,47$ & 0,524 \\
\hline Diarrea & 0,74 & $0,29-1,90$ & 0,541 \\
\hline \multicolumn{4}{|l|}{ Comorbilidades } \\
\hline Hipertensión arterial & 0,98 & $0,19-4,78$ & 0,986 \\
\hline Diabetes mellitus & 0,37 & $0,07-1,89$ & 0,224 \\
\hline Obesidad & 1,84 & $0,78-4,65$ & 0,170 \\
\hline \multicolumn{4}{|l|}{ Terapia } \\
\hline Uso de ventilación mecánica & 8,75 & $4,45-17,22$ & 0,000 \\
\hline
\end{tabular}


En el análisis multivariado, la edad de 60 años a más $(O R=4,74)$, la dificultad respiratoria $(O R=2,38)$ y el uso de ventilación mecánica $(O R=8,75)$ estuvieron asociados a mayor riesgo de mortalidad (Tabla 5).

Tabla 5. Factores asociados en pacientes fallecidos con covid-19 (análisis multivariado)

\begin{tabular}{|c|c|c|c|}
\hline Variable & $\begin{array}{l}\text { Odds ratio } \\
\text { (OR) }\end{array}$ & IC a $95 \%$ & Valor de $p$ \\
\hline \multicolumn{4}{|l|}{ Aspectos demográficos } \\
\hline Edad $>60$ años & 4,74 & $2,94-7,65$ & 0,000 \\
\hline Sexo masculino & 1,59 & $0,96-2,64$ & 0,071 \\
\hline \multicolumn{4}{|l|}{ Síntomas } \\
\hline Tos & 0,87 & $0,49-1,53$ & 0,633 \\
\hline Malestar general & 0,89 & $0,53-1,49$ & 0,681 \\
\hline Fiebre & 0,38 & $0,23-0,64$ & 0,000 \\
\hline Dificultad respiratoria & 2,38 & $1,42-3,99$ & 0,001 \\
\hline Dolor de garganta & 0,96 & $0,57-1,61$ & 0,886 \\
\hline Cefalea & 0,87 & $0,49-1,56$ & 0,660 \\
\hline Dolor muscular & 0,76 & $0,40-1,43$ & 0,397 \\
\hline Dolor de pecho & 1,13 & $0,59-2,15$ & 0,706 \\
\hline Congestión nasal & 1,24 & $0,63-2,47$ & 0,524 \\
\hline Diarrea & 0,74 & $0,29-1,90$ & 0,541 \\
\hline \multicolumn{4}{|l|}{ Comorbilidades } \\
\hline Hipertensión arterial & 0,98 & $0,19-4,78$ & 0,986 \\
\hline Diabetes mellitus & 0,37 & $0,07-1,89$ & 0,224 \\
\hline Obesidad & 1,84 & $0,78-4,65$ & 0,170 \\
\hline \multicolumn{4}{|l|}{ Terapia } \\
\hline Uso de ventilación mecánica & 8,75 & $4,45-17,22$ & 0,000 \\
\hline
\end{tabular}

\section{DISCUSIÓN}

La COVID-19 es una enfermedad respiratoria aguda con repercusión sistémica, altamente transmisible y que es causada por el coronavirus del síndrome respiratorio agudo severo (SARS-CoV-2) ${ }^{(14)}$. La pandemia de la enfermedad de la COVID-19 está ocasionando un gran perjuicio en las personas y en la sociedad; la respuesta conductual de la población cumple un papel importante en mitigar la propagación de la enfermedad ${ }^{(15)}$. Según algunos estudios, el promedio de edad de los pacientes con COVID-19 oscila entre 45,7 y 55,6 años ${ }^{(16-18)}$, y la frecuencia en hombres está entre entre $57,3 \%$ y $72 \%{ }^{(16-20)}$. En nuestra serie encontramos un promedio de edad de 49 años, y mayor frecuencia de presencia de la enfermedad en hombres que en mujeres.

La prevalencia de la COVID-19 por sexo difiere entre los países. En España, al inicio de la pandemia, fue más frecuente en los hombres y luego, en las mujeres; a fines de abril del 2020, la mortalidad era la misma en hombres $(67 \%)$ que en mujeres $(66 \%)^{(21)}$. Otros reportes muestran que los hombres experimentan una mayor gravedad y mortalidad por la COVID-19 (3,22-25). Las mujeres son más vulnerables a la infección por el SARS-CoV-2 entre los 30 y 60 años y la tasa de letalidad es más alta en hombres (hombres/mujeres entre 1,4 y 1,7$)^{(26)}$. En nuestra serie, la COVID-19 fue más frecuente en hombres que en mujeres, tanto en la prevalencia como en la mortalidad. Para explicar las diferencias atribuidas al sexo, existen múltiples factores de tipo genético, hormonal, inmunológico, molecular, social, conductual y de estilos de vida ${ }^{(24,25,27)}$.

Los datos sugieren que los hombres sufren cuadros más severos que las mujeres, porque las comorbilidades asociadas a la COVID-19, como la hipertensión arterial, la enfermedad cardiovascular y la enfermedad pulmonar, son más prevalentes en ellos y, en muchos casos, se relacionan al hábito de fumar y al consumo de alcohol ${ }^{(28)}$. Los aspectos inmunológicos explican esta diferencia. Asimismo, se considera que las mujeres tienen, aproximadamente, un $50 \%$ más de probabilidades que los hombres de adoptar y practicar comportamientos adecuados de bioseguridad 
(lavarse las manos, usar mascarillas y evitar el transporte público) ${ }^{(15)}$.

El periodo de incubación de la enfermedad fue de 5 días, en promedio; en todos los casos las manifestaciones se desarrollaron en los 12 días posteriores a la infección ${ }^{(22,23,29)}$. Los síntomas de la COVID-19 son extremadamente heterogéneos y varían de cuadros muy leves a de extrema gravedad ${ }^{(23,30)}$. Los adultos mayores presentan un mayor riesgo de enfermarse de forma severa ${ }^{(30)}$, con hipoxia significativa y síndrome de distress de respiratorio ${ }^{(23)}$.

Las principales manifestaciones clínicas de la COVID-19 son la fiebre (44\% a $98 \%$ ), tos (69 \% a $82 \%$ ), disnea (19\% a $64 \%$ ), rinorrea $(4 \%$ a $24 \%$, dolor de garganta (5 \% a $14 \%$ ), cefalea (5 \% a $10 \%)$, diarrea (2 \% a $5 \%$ ), náusea y/o vómitos $(1 \%$ a $5 \%$ ) y mialgia $(11 \% \text { a } 15 \%)^{(7,17,19,31-36)}$. También se describen perdida del olfato $(40,0 \%)$ y del gusto $(23,8 \%)^{(17,37)}$. En nuestra serie los síntomas más frecuentes fueron similares a lo reportado: los pacientes presentaron tos (70,26\%), malestar general $(57,63 \%)$, disnea $(48,81 \%)$, fiebre $(48,15 \%)$, dolor de garganta $(44,21 \%)$, cefalea $(26,44 \%)$, mialgia $(23,68 \%)$, diarrea $(11,31 \%)$ y náusea y/o vómitos $(9,21 \%)$. Algunos estudios señalan que los pacientes graves y críticos pueden presentar una fiebre baja o incluso no presentarla, lo que podría evidenciar un sistema inmunológico disminuido o deficiente ${ }^{(6,23)}$, característica que también se pudo observar en nuestro estudio. Otro dato importante de nuestra casuística fue la disnea, que estaría relacionada con un daño pulmonar ocasionado por la COVID-19, provocando inadecuada oxigenación (23).

Tian et al. reportaron que el $73,3 \%$ de los casos estudiados eran leves; el 17,6\%, graves; y el 5,0\% eran asintomáticos ${ }^{(22)}$. En nuestro estudio, casi la mitad de los casos fueron leves, y el 7,36 \% fueron casos críticos. Los enfermos con cuadros leves se recuperaron en 2 semanas, aproximadamente; mientras que los cuadros severos o graves mejoraron en 3 a 6 semanas.

La presencia de comorbilidades asociadas a la COVID-19 oscila entre $16 \%$ y 50,5\%, entre ellas están la obesidad, hipertensión arterial, diabetes, enfermedad cardiovascular y cerebrovascular, cáncer, enfermedad renal crónica, asma, antecedente de tuberculosis e hipotiroidismo ${ }^{(7,16,19,38-40)}$. En los pacientes con COVID-19, la prevalencia de hipertensión arterial está entre 4,5\% y $30 \%$ (según el grupo etario, y se incrementa con la edad); la diabetes, entre $5 \%$ y $32 \%$; la enfermedad cardiovascular, entre $1 \%$ y $18 \%{ }^{(8,16,35,41,42)}$; la obesidad, en $42,5 \%{ }^{(40)}$; y el asma, en $6,5 \%{ }^{(40)}$. En nuestra serie, las comorbilidades mostraron una baja frecuencia. Los antecedentes reportan que el $40 \%$ de los enfermos requirió hospitalización, y en el $8 \%$ necesitó internarse en la unidad de cuidados intensivos ${ }^{(20)}$. Nosotros encontramos que casi la mitad de los pacientes fueron hospitalizados y $7,36 \%$ ingresaron en cuidados intensivos.

La frecuencia de mortalidad varía entre $2,3 \%$ y $12,8 \%$ en diferentes países ${ }^{(8,20)}$, se incrementa al aumentar la edad del paciente, por lo que es un predictor de mortalidad, sobre todo para los hombres ${ }^{(43)}$. La mortalidad es el marcador más simple de vulnerabilidad ante la COVID-19 (43) y está incrementada en pacientes con comorbilidades ${ }^{(20,43)}$. Asimismo, a medida que aumenta la edad, se observan formas más graves de enfermedad y con un riesgo mayor de mortalidad a partir de los 55 años ${ }^{(44)}$, situación muy similar a nuestra casuística.

Se ha reportado que la altitud podría disminuir la tasa de infección y la mortalidad por la COVID-19 ${ }^{(45,46)}$. De acuerdo a Segovia-Juárez et al., a una gran altura, la infección por COVID-19 se reduce, mientras que la tasa de letalidad no depende de la altitud; además, no reportan diferencias significativas entre la frecuencia de letalidad entre varones y mujeres, según niveles de altitud ${ }^{(47)}$. Cano-Pérez et al. sugieren que las grandes altitudes pueden inducir una disminución en la tasa de letalidad de la COVID-19 (48). Por otro lado, Wolcott et al. han reportado un incremento de la mortalidad a más de $2000 \mathrm{~m} \mathrm{~s}$. n. m. en varios condados de Estados Unidos de Norteamérica, así como en México, en comparación con lugares ubicados por debajo de los 1500 $\mathrm{m} \mathrm{s}$. n. m., lo que es más frecuente en hombres mexicanos menores de 65 años ${ }^{(49)}$. Encontramos una tasa de letalidad más alta en los varones a partir de 50 años; sin embargo, en el análisis multivariado no hubo diferencia significativa entre varones y mujeres.

Existen factores de riesgo relacionados a la letalidad en la COVID-19 tales como la diabetes de inicio temprano, obesidad, enfermedad pulmonar obstructiva, edad avanzada, hipertensión arterial, inmunosupresión y enfermedad renal crónica ${ }^{(50)}$. Se ha reportado un incremento de la mortalidad con la edad avanzada $(O R=2,15)$, sexo masculino $(O R=1,97)$ e historia de diabetes $(O R=2,34){ }^{(51)}$. Zheng et al. reportan varios factores de riesgo asociados al pronóstico de la COVID-19 como hipertensión $(O R=2,72)$, diabetes $(O R=3,25)$, enfermedad cardiovascular $(O R=5,19)$ y enfermedad respiratoria $(O R=5,15)$. Asimismo, la edad mayor a 65 años $(O R=6,01)$, el sexo masculino $(O R=1,77)$ y el consumo de tabaco $(O R=2,04)$ se relacionan a un mayor riesgo de desarrollar una enfermedad severa ${ }^{(52)}$. La disnea $(\mathrm{OR}=4,16)$ se asocia a la progresión de la enfermedad y a una elevada mortalidad ${ }^{(52,53)}$, mientras que la presencia de fiebre $(O R=0,56)$ puede traducir una protección al paciente con COVID-19 para evitar el desarrollo de un cuadro grave ${ }^{(52)}$. En nuestra serie encontramos hallazgos similares respecto a la disnea $(O R=2,38)$ y la fiebre $(O R=0,38)$.

Las limitaciones de este estudio fueron la falta de seguimiento de los pacientes con diagnóstico de COVID-19 por un periodo más prolongado, no haber contado con 
un grupo de estudio comparativo en ciudades situadas a menor altitud y no emplear las pruebas moleculares para identificar a los pacientes enfermos.

Se concluye que la enfermedad de la COVID-19 es más frecuente en los hombres que en las mujeres, y los grupos más afectados por esta enfermedad son las amas de casa y los comerciantes. Las características clínicas más frecuentes fueron tos, malestar general, fiebre, dificultad respiratoria y dolor de garganta. La obesidad, hipertensión arterial y diabetes mellitus fueron las comorbilidades más comunes, pero se encontraban en un porcentaje bajo; mientras que la edad avanzada, la dificultad respiratoria y el uso de ventilación mecánica se asociaron a mayor riesgo de letalidad. El mejor método de control de la propagación de la enfermedad es la prevención de la infección, la detección precoz del virus y la identificación de protocolos de tratamientos exitosos.

Contribuciones de autoría: ADL participó en el diseño del estudio, recolección de datos, análisis e interpretación de los datos, en la redacción del manuscrito y aprobó la versión final. RMO se encargó del diseño del estudio, recolección de los datos, interpretación de los datos y aprobó la versión final. ELN participó en el diseño del estudio, interpretación de los datos y aprobó la versión final. EAL estuvo a cargo del diseño, recolección de datos y aprobó la versión final.

Fuentes de financiamiento: Este artículo ha sido financiado por los autores.

Conflicto de interés: Los autores declaran no tener conflicto de intereses.

\section{REFERENCIAS BIBLIOGRÁFICAS}

1. Jiang F, Deng L, Zhang L, Cai Y, Cheung CW, Xia Z. Review of the clinical characteristics of coronavirus disease 2019 (COVID-19). J Gen Intern Med. 2020; 35(5): 1545-9.

2. Yan-Rong G, Qing-Dong C, Zhong-Si H, Yuan-Yang T, Shou-Deng C, Hong-Jun J, et al. The origin, transmission, and clinical therapies on coronavirus disease 2019 (COVID-19) outbreak - an update on the status. Mil Med Res. 2020; 7(1): 11.

3. Gebhard C, Regitz-Zagrosek V, Neuhauser HK, Morgan R, Klein SL. Impact of sex and gender on COVID-19 outcomes in Europe. Biol Sex Differ. 2020; 11: 29.

4. Pascarella G, Strumia A, Piliego C, Bruno F, Buono RD, Costa F, et al. COVID-19 diagnosis and management: a comprehensive review. J Intern Med. 2020; 288(2): 192-206.

5. Shi Y, Wang G, Cai X-P, Deng J-W, Zheng L, Zhu H-H, et al. An overview of COVID-19. J Zhenjiang Univ Sci B. 2020; 21(5): 343-60.

6. Li H, Liu S-M, Yu X-H, Tang S-L, Tang C-K. Coronavirus disease 2019 (COVID-19): current status and future perspectives. Int J Antimicrob Agents. 2020; 55(5): 105951.

7. Ge H, Wang X, Yuan X, Xiao G, Wang Deng T, et al. The epidemiology and clinical information about COVID-19. Eur J Clin Microbiol Infect Dis. 2020; 39(6): 1011-9.

8. Tadic M, Cuspidi C, Mancia G, Dell'Oro R, Grassi G. COVID-19, hypertension and cardiovascular diseases: Should we change the therapy?. Pharmacol Res. 2020; 158: 104906.

9. Ministerio de Salud. Alerta epidemiológica ante riesgo de intensificación de la transmisión comunitaria de COVID-19 en el periodo post cuarentena, en el Perú [Internet]. Lima; 2020. Disponible en: https://www.dge.gob.pe/portal/docs/ alertas/2020/AE019.pdf

10. Hoffman T, Nissen K, Krambich J, Rönnberg B, Akaberi D, Esmaeilzadeh M, et al. Evaluation of a COVID-19 IgM and IgG rapid test; an efficient tool for assessment of past exposure to SARS-CoV-2. Infect Ecol Epidemiol. 2020; 10(1): 1754538.

11. Instituto Nacional de Estadística e Informática. Perú: factores de riesgo asociados a complicaciones por COVID-19. ENDES 20182019 [Internet]. Lima: 2020. Disponible en: https://www.inei. gob.pe/media/MenuRecursivo/publicaciones_digitales/Est/ Lib1738/libro.pdf

12. Organización Panamericana de la Salud. Definiciones de casos para la vigilancia COVID-19 - 16 de diciembre del 2020 [Internet]. OPS; 2020. Disponible en: https://www.paho.org/es/temas/ coronavirus/brote-enfermedad-por-coronavirus-covid-19/ definiciones-casos-para-vigilancia

13. Escobar G, Matta J, Ayala R, Amado J. Características epidemiológicas de pacientes fallecidos por COVID-19 en un Hospital Nacional de Lima, Peru. Rev Fac Med Hum. 2020; 20(2): 180-5.

14. Tenda ED, Yulianti M, Asaf MM, Yunus RE, Septiyanti W, Wulani $\mathrm{V}$, et al. The importance of chest CT Scan in COVID-19. Acta Med Indones. 2020: 52(1): 68-73.

15. Moran KR, Del Valle SY. A meta-analysis of the association betwween gender and protective behaviors in response to respiratory epidemics and pandemics. PLoS One. 2016; 11(10): e0164541.

16. Guan W-J, Liang W-H, Zhao Y, Liang H-R, Chen Z-S, Li Y-M, et al. Comorbidity and its impact on 1590 patients with COVID-19 in China: a nationwide analysis. Eur Respir J. 2020; 55(5): 2000547.

17. Yusuf MMF, Garad MY, Mohamed AA, Adam AB. Loss of taste and smell are common clinical characteristics of patients with COVID-19 in Somalia: A retrospective double centre study. Infec Drug Resist. 2020; 13: 2631-5.

18. Zhang J, Wang X, Jia X, Li J, Hu K, Chen G, et al. Risk factors for disease severity, unimprovement, and mortality in COVID-19 patients in Wuhan, China. Clin Microbiol Infect. 2020; 26(6): 767-72.

19. Guzman-Del Giudice OE, Lucchesi-Vásquez EO, Trelles-De Belaúnde M, Pinedo-Gonzales RH, Daly A, et al. Características clínicas y epidemiológicas de 25 casos de COVID-19 atendidos en la Clínica Delgado de Lima. Rev Soc Per Med Interna. 2020; 33(1): 15-24.

20. Zhang G, Hu C, Luo L, Fang F, Chen Y, Li J, et al. Clinical features and short-term outcoms of 221 patients with COVID-19 in Wuhan, China. J Clin Virol. 2020; 127: 104364.

21. Cantero MTR. Las estadísticas sanitarias y la invisibilidad por sexo y de género durante la epidemia de COVID-19. Gac Sanit. 2021; 35(1): 95-8

22. Mukherjee S, Pahan K. Is COVID-19 Gender-sensitive?. J Neuroimmune Pharmacol. 2021; 1-10.

23. Gadi N, Wu SC, Spihlman AP, Moulton VR. What's sex got to do with COVID-19? Gender-based differences in the host immune response to coronaviruses. Front Immunol. 2020; 11: 2147.

24. Mangia C, Russo A, Civitelli S, Gianicolo EAL. Sex/gender differences in COVID-19 lethality: what the data say, and do not say. Epidemiol Prev. 2020; 44 (5-6 Suppl. 2): 400-6.

25. Pradhan A, Olsson P-E. Sex differences in severity and mortality from COVID-19: are males more vulnerable?. Biol Sex Differ. 
2020; 11(1): 53.

26. Hall KS, Samari G, Garbers S, Casey SE, Diallo DD, Orcutt M, et al. Centring sexual and reproductive health and justice in the global COVID-19 response. Lancet. 2020; 395(10231): 1175-7.

27. Tian S, Hu N, Lou J, Chen K, Kang X, Xiang Z, et al. Characteristics if COVID-19 infection in Beijing. J Infect. 2020; 80(4): 401-6.

28. Yuki K, Fujiogi M, Koutsogiannaki S. COVID-19 pathophysiology: A review. Clin Immunol. 2020; 215: 108427.

29. Lauer SA, Grantz KH, Bi Q, Jones FK, Zheng Q, Meredith HR, et al. The incubation period of coronavirus disease 2029 (COVID-19) from publicly reported confirmed cases: Estimation and Application. Ann Intern Med. 2020: M20-0504.

30. Frater JL, Zini G, d'Onofrio G, Rogers HJ. COVID-19 and clinical hematology laboratory. Int J Lab Hematol. 2020; 42(Suppl. 1): 11-8.

31. Du R-H, Liang L-R, Yang C-Q, Wang W, Cao T-Z, Li M, et al. Predictors of mortality for patients with COVID-19 pneumonia caused by SARS-CoV-2: a prospective cohort study. Eur Respir J. 2020; 55(5): 2000524.

32. Guan W, Ni Z, Hu Y, Liang W, Ou C, He J, et al. Clinical characteristics of coronavirus disease 2019 in China. N Engl J Med. 2020; 382: 1708-20.

33. Chen N, Zhou M, Dong X, Qu J, Gong F, Han Y, et al. Epidemiological and clinical characteristics of 99 cases of 2019 novel coronavirus pneumonia in Wuhan, China: a descriptive study. Lancet. 2020; 395(10223): 507-13.

34. Shi H, Han X, Jiang N, Cao Y, Alwalid O, Gu J, et al. Radiological findings from 81 patients with COVID-19 pneumonia in Wuhan, China: a descriptive study. Lancet Infect Dis. 2020; 20(4): 425-34.

35. Huang C, Wang Y, Li X, Ren L, Zhao J, Hu Y, et al. Clinical features of patients infected with 2019 novel coronavirus in Wuhan, China. Lancet. 2020; 395(10223): 497-506.

36. Lai CL, Shih TP, Ko WC, Tang HJ, Hsuch PR. Severe acute respiratory syndrome coronavirus 2 (SARS-CoV-2) and Coronavirus disease-2019 (COVID-19): The epidemic and the challenges. Int J Antimicrob Agents. 2020; 55(3): 105924.

37. Kabir T, Uddin S, Hossain F, Abdulhakim J, Alam A, Asrhaf G., et al. nCOVID-19 pandemic: from molecular pathogenesis to potential investigational therapeutics. Front Cell Dev Biol. 2020; 8: 616.

38. Yang C-L, Qiu X, Zeng Y-K, Jiang M, Fan H-R, Zhang Z-M. Coronavirus disease 2019: a clinical review. Eur Rev Med Pharmacol Sci. 2020; 24 (8): 4585-96.

39. Huang C, Wang Y, Li X, Ren L, Zhao J, Hu Y, et al. Clinical features of patients infected with 2019 novel coronavirus in Wuhan, China. Lancet. 2020; 395(10223): 497-506.

40. Mejía F, Medina C, Cornejo E, Morello E, Vásquez S, Alave J, et al. Características clínicas y factores asociados a mortalidad en pacientes adultos hospitalizados por COVID-19 en un hospital público de Lima, Perú. Preprints Scielo. 2020.

41. Yang X, Yu Y, Xu J, Shu H, Xia J, Liu H, et al. Clinical course and outcomes of critically ill patients with SARS-CoV-2 pneumonia in Wuhan, China: a single-centered, retrospective, observational study. Lancet Resp Med. 2020; 8(5): 475-81.

42. Llaro-Sánchez MK, Gamarra-Villegas BE, Campos-Correa KE. Características clínico-epidemiológicas y análisis de sobrevida en fallecidos por COVID-19 atendidos en establecimientos de la Red Sabogal Callao. 2020. Horiz Med. 2020; 20(2): e1229.

43. Blagosklonny M. From causes of aging to death from COVID-19. Aging (Albany NY). 2020; 12(11): 10004-21.

44. Uddin M, Mustafa F, Rizvi TA, Loney T, Suwaidi HA, AL-Marzouqi $\mathrm{AH}$, et al. SARS-CoV-2/COVID-19: Viral genomics epidemiology vaccines and therapeutic interventions. Viruses. 2020; 12(5): 526.
45. Accinelli RA, Leon-Abarca JA. At high altitude COVID-19 is less frequent the experience of Peru. Arch Bronconeumol. 2020; 56(11): 760-1.

46. Quevedo-Ramirez A, Al-Kassab-Cordova A, Mendez-Guerra C, Cornejo-Venegas G, Alva-Chavez KP. Altitude and excess mortality during COVID-19 pandemic in Peru. Respir Physiol Neurobiol. 2020; 281: 103512.

47. Segovia-Juarez J, Castagnetto JM, Gonzales GF. High altitude reduces infection rate of COVID-19 but no case fatality rate. Respir Physiol Neurobiol. 2020; 281: 103494.

48. Cano-Pérez E, Torres-Pacheco J, Fragozo-Ramos MC, García-Díaz G, Montalvo-Varela E, Pozo-Palacios JC. Negative correlation between altitude and the COVID-19 pandemic in Colombia: a preliminary report. Am J Trop Hyg. 2020; 103(6): 2347-9.

49. Woolcott 00, Bergman RN. Mortality attributed to COVID-19 in high altitude populations. High Alt Med Biol. 2020; 21(4): 409-16.

50. Bello-Chavolla OY, Bahena-López JP, Antonio-Villa NE, VargasVásquez A, Gonzáles-Díaz A, Márquez-Salinas A, et al. Predicting mortality due to SARS-CoV-2: A mechanistic score relating obesity and diabetes to COVID-19 outcomes in Mexico. J Clin Endocrinol Metab. 2020; 105(8): dgaa346.

51. Yu C, Lei Q, Li W, Wang X, Liu W, Fan X, et al. Clinical characteristics, associated factors, and predicting COVID-19 mortality risk: A retrospective study in Wuhan, China. Am J Prev Med. 2020; 59(2): 168-75.

52. Zheng Z, Peng F, Xu B, Zhao J, Liu H, Peng J, et al. Risk factors of critical \& mortal COVID-19 cases: A systematic literature review and meta-analysis. J Infect. 2020; 81(2): e16-25.

53. Shi L, Wang $Y$, Wang $Y$, Duan G, Yang H. Dyspnea rather than fever is a risk factor for predicting mortality in patients with COVID-19. J Infect. 2020; 81(4): 647-9.

\section{Correspondencia:}

Anibal Diaz-Lazo

Dirección: Calle Ricardo Palma № 553, Urb. Ingeniería. Lima, Perú.

Teléfono: 51-481 0838.

Correo electrónico: andiaz1612@hotmail.com

Recibido: 18 de septiembre de 2020 Evaluado: 22 de diciembre de 2020 Aprobado: 01 de marzo de 2021

(c) La revista. Publicado por Universidad de San Martín de Porres, Perú. (c) $\mathbf{B Y}$ Licencia de Creative Commons Artículo en acceso abierto bajo términos de Licencia Creative Commons Atribución 4.0 Internacional. (http://creativecommons.org/licenses/by/4.0/)

\section{ORCID iDs}

Aníbal Díaz-Lazo

Raul Montalvo Otivo

Ernesto Lazarte Nuñez

Edinson Aquino Lopez

(1) https: / / orcid.org/0000-0002-9282-9435 (1) https: / / orcid.org/0000-0003-0227-8850 (1) https: / / orcid.org/0000-0002-7025-6998 (ㄱ) https: / / orcid.org/0000-0002-1241-9250 